\title{
Subclinical hypothyroidism in children with well-controlled epilepsy
}

\author{
Ali Akbar Momen ${ }^{1}$, Tahereh Ziaei Kajbaf², Ashraf Sepehran², Majid Aminzadeh²,3, \\ Reza Azizi Malamiri' \\ 'Department of Paediatric Neurology, Golestan Medical, Educational, and Research Centre, Ahvaz Jundishapur University \\ of Medical Sciences, Ahvaz, Iran \\ 2Paediatric Department, Faculty of Medicine, Ahvaz Jundishapur University of Medical Sciences, Ahvaz, Iran \\ ${ }^{3}$ Diabetes Research Centre, Health Research Institute, Ahvaz Jundishapur University of Medical Sciences, Ahvaz, Iran
}

\section{ABSTRACT}

Introduction: Epilepsy and epileptic syndromes are common in children. Frequent seizures have a great negative impact on the quality of life in children and their families, and many children with epilepsy need anticonvulsive therapy.

Aim of the study: Despite reducing seizure frequency, anticonvulsive therapy has many complications. Subclinical hypothyroidism has been argued in children with epilepsy as a complication of anticonvulsive therapy and has been proposed as a risk factor for long-term cardiovascular problems. We aimed to determine the relative frequency of subclinical hypothyroidism in children with well-controlled epilepsy.

Material and methods: In a prospective cohort, we assessed subclinical hypothyroidism in 228 children with epilepsy, who were treated with sodium valproate $(n=93)$, carbamazepine $(n=76)$, and phenobarbital $(n=59)$ as monotherapy and compared them with 100 age- and sex-matched healthy children as controls. We defined subclinical hypothyroidism as serum TSH level more than $3.8 \mu \mathrm{IU} / \mathrm{ml}$ and normal serum T4 level. Results: According to the definition, subclinical hypothyroidism was significantly more frequent in children with epilepsy than in healthy controls. Subclinical hypothyroidism was found in 25 (26.8\%) children in the sodium valproate group, $15(19.7 \%)$ in the carbamazepine group, and 13 (22\%) in phenobarbital group, but only in four (4\%) children in the control group.

Conclusions: These results indicate that in children with epilepsy, who were treated by one of the following: carbamazepine, sodium valproate, or phenobarbital, as monotherapy, subclinical hypothyroidism could occur frequently. Monitoring of thyroid function using T4 and TSH serum level should be considered in these children during anticonvulsive therapy. However, long-term effects of anticonvulsive medications on thyroid function need well-designed, prolonged cohort studies.

\section{KEY WORDS:}

children, epilepsy, subclinical hypothyroidism, anticonvulsive therapy.

\section{ADDRESS FOR CORRESPONDENCE:}

Reza Azizi Malamiri, Department of Paediatric Neurology, Golestan Medical, Educational, and Research Centre, Ahvaz Jundishapur University of Medical Sciences, \# 90, Golestan Blvd., P.O. Box 6135733118, Ahvaz, Iran, e-mail: azizi.ramin@gmail.com 


\section{INTRODUCTION}

Epilepsy is one the most common neurologic disorders in children. Children with infrequent seizures have a better quality of life; therefore, many children with epilepsy need treatment with anticonvulsants to reduce seizure recurrence. First- and second-generation anticonvulsants such as phenobarbital, carbamazepine, and sodium valproate are among the most common anticonvulsants that physicians administer in children with epilepsy. Unfortunately, despite favourable outcomes such as reducing seizure frequency, these anticonvulsants have many adverse side effects. One of the most frequently discussed adverse effects after anticonvulsive administration is thyroid function disturbances and probable subclinical hypothyroidism [1-11].

Subclinical hypothyroidism has been defined by increased thyroid-stimulating hormone (TSH) serum levels while serum levels of T4 and T3 are normal. Subclinical hypothyroidism could lead to long-term complications such as cardiovascular problems. According to this evidence, some of the investigators claimed to find and, if required, to treat subclinical hypothyroidism in patients with chronic conditions to prevent such long-term complications [12-14].

In our country, Iran, many paediatricians and child neurologists administer phenobarbital, carbamazepine, and sodium valproate to manage children with epilepsy. To our knowledge, no study with an acceptable sample size has been conducted to evaluate subclinical hypothyroidism in children with epilepsy, who are treated with these anticonvulsants in our country. Therefore, we conducted a study using an acceptable sample size to assess the frequency of subclinical hypothyroidism in children with epilepsy, who were treated with phenobarbital, carbamazepine, and sodium valproate. We also tried to show a relation between these anticonvulsants and the degree of subclinical hypothyroidism.

\section{MATERIAL AND METHODS}

\section{STUDY LOCATION}

We conducted this study at Golestan Hospital, a major tertiary university-affiliated centre of child neurology in the south-west of Iran. This centre serves all the population of the Khuzestan province and five other neighbouring provinces. Almost all of the children with epilepsy in these provinces were referred to this centre for better evaluation and treatment. All of these children were evaluated and treated under direct supervision of three child neurologists from the centre. One of these child neurologists has a subspecialty in epileptology and clinical neurophysiology. The study was started in April 2014 and was continued to reach the predetermined sample size by March 2018.

\section{PARTICIPANTS, INCLUSION, AND EXCLUSION CRITERIA}

All children with generalised or focal epilepsy according to the International League Against Epilepsy (ILAE) classification [15] were potentially eligible to recruit. To reduce the probable effect of using more than two anticonvulsive medications on the thyroid function tests in a patient, we included only those children with epilepsy, who were treated with one of the following: phenobarbital, or carbamazepine, or sodium valproate generic forms as monotherapy. To reduce the effect of high dosages of anticonvulsive medications on the thyroid function tests, we administered anticonvulsive medications at standard maintenance dosages (phenobarbital $5 \mathrm{mg} / \mathrm{kg} / \mathrm{dose}$ at bedtime, carbamazepine $10-15 \mathrm{mg} / \mathrm{kg} /$ dose every $12 \mathrm{~h}$, and sodium valproate $10-15 \mathrm{mg} / \mathrm{kg} /$ dose every $12 \mathrm{~h}$ ). All the children with epilepsy were well-controlled using these dosages. We excluded all children who had one of the following criteria: 1) uncontrolled epilepsy [16] (more than one seizure event in the last six months) because children with uncontrolled epilepsy need frequent changes in anticonvulsant dosages and even changes in their anticonvulsants, and these changes may have additive effects on thyroid function; 2) anticonvulsive treatment for less than six months; 3 ) treatment with more than two anticonvulsive agents; 4 ) a chronic condition other than epilepsy such as cardiovascular, endocrine, renal, and hepatic disease, global developmental delay or confirmed chromosomal abnormalities, and taking medications other than anticonvulsants, because all of these conditions may affect the thyroid function tests; and 5) confirmed thyroid disorders. We also assessed a group of age- and sex-matched children as a control group. We selected these controls from the children who were referred for growth and development monitoring and were residents of the same area as the patients. Many of the children in the control group were siblings of the children with epilepsy.

\section{DESIGN}

At least two child neurologists (one of them has a subspecialty in epileptology and clinical neurophysiology) visited all the children with epilepsy and interviewed all the parents and their children seeking medical history for risk factors of epilepsy. When video clips of the events were available, these child neurologists thoroughly reviewed these clips to determine the seizure type. After taking a thorough history of the event, reviewing the video clips, examining the children, reviewing the brain MRI when available, and reviewing the EEG findings, these two child neurologists decided about the epilepsy syndrome according to the ILAE classification [15] if possible. Of note, many of the children with epilepsy were referred to our child neurology clinic for a better decision 
and follow-up. After visiting these children with epilepsy, we tried to define the epileptic syndrome and to decide about selecting and administering the best anticonvulsive medication. A child endocrinologist thoroughly interviewed and examined all the participants for the clinical manifestations of hypothyroidism.

We registered the following data from the participants: age, sex, body weight, height, body mass index, seizure type and if possible epilepsy syndrome according to the ILAE classification, EEG and neuroimaging findings, and duration of anticonvulsive therapy.

We obtained informed consent from all the participants and their parents before blood sampling. We referred all the participants to the central endocrine laboratory of the centre for blood sampling between 8:00 and 10:00 a.m. every day except Friday. In each participant, a 5-ml blood sample was collected under standard aseptic conditions. All the samples were stored at $-20^{\circ} \mathrm{C}$ until time of analysis. A thyroid function test (T3, T4, and TSH) was performed for each participant using Thyroid RIA kits (Padyab Teb Co. Apt. 8, No. 17, Eastern Shab boo Aly., 1st Eastern St., Saadat Abad Ave, Tehran, Iran, Telefax: (+9821) 26417197). The normal reference ranges of the thyroid function tests in our area were: TSH (0.2-3.8 $\mu \mathrm{IU} / \mathrm{ml}), \mathrm{T} 4(45-160 \mu \mathrm{g} / \mathrm{dl})$, and T3 (1.2-3.1 ng/ml) [17]. We defined subclinical hypothyroidism as a serum T4 within the normal range and increased TSH serum levels above the normal ranges. We also graded subclinical hypothyroidism as grade I (TSH 3.9-6 $\mu \mathrm{IU} / \mathrm{ml}$ ), grade II (TSH 6.1-10 $\mu \mathrm{IU} / \mathrm{ml}$ ), and grade III (TSH $>10 \mu \mathrm{IU} / \mathrm{ml}$ ). [9] To define the precision in assessing thyroid function tests, we measured both intra-assay and inter-assay coefficient of variation for all the thyroid function tests (T3, $\mathrm{T} 4$, and TSH). The T4 levels had an intra-assay coefficient of variation $<3.1 \%$ and an inter-assay coefficient of variation $<2.5 \%$. The T3 levels had an intra-assay coefficient of variation $<5.1 \%$ and an inter-assay coefficient of variation $<6 \%$. The TSH levels had an intra-assay coefficient of variation $<5 \%$ and an inter-assay coefficient of variation $<6.4 \%$. In each participant, thyroid function tests were conducted once; however, if the results of thyroid function tests were abnormal, we repeated them after four weeks to confirm that the participant definitely had subclinical hypothyroidism.

\section{STATISTICS}

We calculated the sample size by using a study conducted by Aggarwal et al. [1] To define the differences of thyroid function tests between these three groups of children with epilepsy treated with anticonvulsive monotherapy against control group and for a type I error of 0.05 and type II error of 0.2 , we needed to have at least 50 children in each treatment group. We collected all data from the participants in a central computerised registry. We described all data using appropriate descriptive statistics. All data were analysed for normality. To compare subgroups of patients and patients against controls we used ANOVA or an appropriate nonparametric equivalent as required. We considered $p$-values less than 0.05 as significant. A biostatistician who was blinded to the treatment groups analysed the data.

\section{ETHICS}

The review board of the centre approved the study protocol, and we performed the study in accordance with the ethical standards as laid down in the 1964 Declaration of Helsinki and its later amendments. All the parents read and signed written, informed consents before any blood sampling.

\section{RESULTS}

\section{BASELINE CHARACTERISTICS}

We studied 228 children with epilepsy and 100 healthy children. All children with epilepsy were thoroughly interviewed and examined to define seizure type and, if possible, to define the epilepsy syndrome. From 228 children with epilepsy, 93 received sodium valproate, 76 received carbamazepine, and 59 received phenobarbital. Table 1 shows the baseline characteristics of the two groups.

From 228 children with epilepsy 100 (43.9\%) children had generalised seizures and $128(56.1 \%)$ children had fo-

TABLE 1. The baseline characteristics of the two groups

\begin{tabular}{|l|c|c|}
\hline & Children with epilepsy $(n=228)$ & Healthy children $(n=100)$ \\
\hline Age in year, median (range) & $7.5(3-15)$ & $7(3-15)$ \\
\hline Sex & & $51(51)$ \\
\hline Boys (\%) & $140(61.4)$ & $49(49)$ \\
\hline Girls (\%) & $88(38.6)$ & $23(13-70)$ \\
\hline Weight in kg, median (range) & $24(11.5-95)$ & $118(95-164)$ \\
\hline Height in cm, median (range) & $125(80-170)$ & $15.36(11.16-29.52)$ \\
\hline BMI, median (range) & $17.09(10.74-37.11)$ & \\
\hline
\end{tabular}


TABLE 2. Thyroid function in children with epilepsy and healthy children

\begin{tabular}{|c|c|c|c|}
\hline & Children with epilepsy $(n=228)$ & Healthy children $(n=100)$ & $p$-value $<0.05^{\mathrm{a}}$ \\
\hline T4 in $\mu \mathrm{g} / \mathrm{dl}$; median (range) & $\begin{array}{l}\text { Phenobarbital }(n=59) ; 82(47-141) \\
\text { Valproate }(n=93) ; 91(55-212) \\
\text { Carbamazepine }(n=76) ; 70(40-148)\end{array}$ & $83.5(30-169)$ & $\begin{array}{l}\text { No } \\
\text { Yes } \\
\text { Yes }\end{array}$ \\
\hline $\mathrm{T} 3$ in ng/ml; median (range) & $\begin{array}{c}\text { Phenobarbital }(n=59) ; 2(0.4-3.1) \\
\text { Valproate }(n=93) ; 2(0.7-3.5) \\
\text { Carbamazepine }(n=76) ; 1.8(0.9-3.6)\end{array}$ & $1.95(0.7-2.4)$ & $\begin{array}{l}\text { No } \\
\text { No } \\
\text { No }\end{array}$ \\
\hline TSH in $\mu \mathrm{IU} / \mathrm{ml}$; median (range) & $\begin{array}{l}\text { Phenobarbital }(n=59) ; 2.9(0.4-7) \\
\text { Valproate }(n=93) ; 3(0.56-12.3) \\
\text { Carbamazepine }(n=76) ; 2.9(0.8-6)\end{array}$ & $2.1(0.4-7.9)$ & $\begin{array}{l}\text { Yes } \\
\text { Yes } \\
\text { Yes }\end{array}$ \\
\hline
\end{tabular}

${ }^{a}$ p-values using Kruskal-Wallis One-Way Analysis of Variance on Ranks

TABLE 3. Thyroid function in boys with epilepsy and healthy boys

\begin{tabular}{|c|c|c|c|}
\hline & Sex & Healthy boys $(n=51)$ & $p$-value $<0.05^{\mathrm{a}}$ \\
\hline T4 in $\mu \mathrm{g} / \mathrm{dl}$; median (range) & $\begin{array}{l}\text { Phenobarbital }(n=39) ; 80(47-115) \\
\text { Valproate }(n=60) ; 90(55-212) \\
\text { Carbamazepine }(n=41) ; 70(40-148)\end{array}$ & $80(30-169)$ & $\begin{array}{l}\text { No } \\
\text { Yes } \\
\text { No }\end{array}$ \\
\hline T3 in ng/ml; median (range) & $\begin{array}{c}\text { Phenobarbital }(n=39) ; 2(0.4-3.1) \\
\text { Valproate }(n=60) ; 2(1-3.5) \\
\text { Carbamazepine }(n=41) ; 1.9(1-3.4)\end{array}$ & $2(1-2.4)$ & $\begin{array}{l}\text { No } \\
\text { No } \\
\text { No }\end{array}$ \\
\hline TSH in $\mu \mathrm{IU} / \mathrm{ml}$; median (range) & $\begin{array}{c}\text { Phenobarbital }(n=39) ; 3(0.4-7) \\
\text { Valproate }(n=60) ; 3(0.5-12) \\
\text { Carbamazepine }(n=41) ; 2.8(0.8-6)\end{array}$ & $2.2(0.4-3.8)$ & $\begin{array}{l}\text { Yes } \\
\text { Yes } \\
\text { No }\end{array}$ \\
\hline
\end{tabular}

a p-values using Kruskal-Wallis One-Way Analysis of Variance on Ranks

TABLE 4. Thyroid function in girls with epilepsy and healthy girls

\begin{tabular}{|l|c|c|c|}
\hline & Girls with epilepsy $(n=88)$ & Healthy girls $(n=49)$ & $p$-value $<0.05^{\text {a }}$ \\
\hline T4 in $\mu \mathrm{g} / \mathrm{dl}$; median (range) & Phenobarbital $(n=20) ; 84(54-141)$ & $84(55-140)$ & No \\
& Valproate $(n=33) ; 91(70-143)$ & No \\
& Carbamazepine $(n=35) ; 70(48-140)$ & & Yes \\
\hline T3 in $\mathrm{ng} / \mathrm{ml}$; median (range) & Phenobarbital $(n=20) ; 1.9(1.2-2.9)$ & $1.9(0.7-2.3)$ & No \\
& Valproate $(n=33) ; 2(0.7-3)$ & & No \\
& Carbamazepine $(n=35) ; 1.8(0.9-3.6)$ & & No \\
\hline TSH in $\mu \mathrm{lU} / \mathrm{ml}$; median (range) & Phenobarbital $(n=20) ; 2.7(1.2-7)$ & $2(0.7-7.9)$ & No \\
& Valproate $(n=33) ; 2.3(0.6-8.8)$ & & No \\
& Carbamazepine $(n=35) ; 3(1.4-5.9)$ & & No \\
\hline
\end{tabular}

${ }^{a} p$-values using Kruskal-Wallis One-Way Analysis of Variance on Ranks

cal seizures. We could define Rolandic epilepsy spectrum in 40 children, generalised tonic-clonic seizures alone in 10 children, childhood absences in eight children, juvenile absences in nine children, juvenile myoclonic epilepsy in 11, and frontal lobe epilepsy in three children. We could not define an epilepsy syndrome in the rest of the children with epilepsy (147 children). From 228 children with epilepsy, $102(44.7 \%)$ had a family history of epilepsy.

From 228 children with epilepsy, 156 children (68.4\%) had abnormal epileptiform findings in their EEG, and in the rest, the EEG findings were normal, especially the background rhythm. From 228 children with epilepsy, brain MRI (non-epilepsy protocol) was conducted in only
175 and the main MRI findings were arachnoid cyst in four children, cavum vergae in six children, colpocephaly in two children, benign external hydrocephaly in four children, and probable focal cortical dysplasia in three children. The brain MRI was normal in the rest of the studies (156 children).

Table 2, Table 3, and Table 4 show thyroid function in children with epilepsy and compare them with healthy children according to the anticonvulsive agents and sex of the participants.

We defined subclinical hypothyroidism as serum TSH level more than $3.8 \mu \mathrm{IU} / \mathrm{ml}$ and normal serum T4 level [17]. According to this definition, 25 (26.8\%) children in the sodium valproate group, 15 (19.7\%) in the carbamaz- 
TABLE 5. The frequency of subclinical hypothyroidism in the participants according to sex and anticonvulsant

\begin{tabular}{|l|c|c|c|c|c|}
\hline Sex and grade & $\begin{array}{c}\text { Valproate } \\
(n=25)\end{array}$ & $\begin{array}{c}\text { Carbamazepine } \\
(n=15)\end{array}$ & $\begin{array}{c}\text { Phenobarbital } \\
(n=13)\end{array}$ & $\begin{array}{c}\text { Healthy } \\
(n=4)\end{array}$ & $\begin{array}{c}p \text {-value } \\
<0.05^{\text {a }}\end{array}$ \\
\hline Boys & 17 & 5 & 7 & 6 & 1 \\
\hline Grade 1 & 8 & 5 & 1 & 0 & Yes \\
\hline Grade 2 & 8 & 0 & 0 & 0 & Yes \\
\hline Grade 3 & 1 & 0 & 4 & 3 & 2 \\
\hline Grads 1 & 8 & 10 & 2 & 1 & 0 \\
\hline Grade 2 & 1 & 10 & 0 & & \\
\hline
\end{tabular}

${ }^{a} \chi^{2}$ tests between anticonvulsive agents and the healthy children

epine group, 13 (22\%) in the phenobarbital group, and only four (4\%) children in the control group had subclinical hypothyroidism. Table 5 shows the frequency and the grade of hypothyroidism in different groups of the participants and compares them.

Subclinical hypothyroidism was significantly $(p<0.05)$ more frequent in children who took anticonvulsive agents than in the healthy controls; however, the frequency of subclinical hypothyroidism among groups of anticonvulsive agents was not significantly different. Of note, none of the children with epilepsy or the healthy controls showed clinical manifestations of hypothyroidism.

\section{DISCUSSION}

Our results show that, compared to healthy children, subclinical hypothyroidism as defined by normal T4 and elevated TSH (more than $3.8 \mu \mathrm{IU} / \mathrm{ml}$ ) [17] was significantly more common in children with epilepsy (more than 23\%, $53 / 228$ ), who were treated using either sodium valproate, carbamazepine, or phenobarbital as monotherapy. For better assessment, we found subclinical hypothyroidism in only $4 \%$ of healthy controls. Despite the relatively high frequency of subclinical hypothyroidism in children with epilepsy, none of the children among those with epilepsy and healthy controls showed clinical signs of hypothyroidism.

According to Table 5, in children who were treated by sodium valproate, subclinical hypothyroidism was more common in boys than in girls (17 boys vs. eight girls). In children who were treated with carbamazepine, subclinical hypothyroidism was more common in girls than in boys (five boys vs. 10 girls), and in those who were treated with phenobarbital, the frequency of subclinical hypothyroidism was the same in boys and in girls. We have no explanation for these differences; however, these results may be accidental, and precisely designed studies in the future could explain these differences.

Our results are in accordance with some of the previous studies. One of the main studies conducted by Eiris-Punal et al. showed that subclinical hypothyroidism could oc- cur in $8.2 \%$ of children with epilepsy, who were treated with carbamazepine (61 children) and $26 \%$ of children who were treated with sodium valproate ( 51 children), compared to $3.6 \%$ (148) of healthy controls [4]. A study conducted by Kim et al. compared 61 children with epilepsy, who were treated with sodium valproate, with 144 healthy controls. They found that $52.4 \%$ (32/61) of children with epilepsy had subclinical hypothyroidism compared to healthy controls $(16.7 \%, 24 / 144)$ [8]. Another study conducted by Turan et al. showed that subclinical hypothyroidism was more common in children who were treated with sodium valproate $(39.2 \%, 20 / 51)$ compared to those who were treated with carbamazepine $(6.7 \%, 3 / 45)$ and phenobarbital $(12.5 \%, 6 / 48)$, and only $2.3 \%(1 / 44)$ of healthy controls showed subclinical hypothyroidism [10]. Another study conducted by Sahu et al., showed subclinical hypothyroidism in 26.3\% (15/57) of children who were treated by sodium valproate as monotherapy [9]. However, two studies conducted by Verroti et al. found that in children with epilepsy, carbamazepine and sodium valproate could alter thyroid function tests; however, interestingly, none of their children showed subclinical hypothyroidism $[18,19]$.

A group of studies has tried to explain the mechanisms of subclinical hypothyroidism in patients treated with anticonvulsants. The main mechanism that has been proposed is GABA ( $\gamma$ aminobutyric acid) stimulating properties of anticonvulsive medications. GABA could inhibit the release of somatostatin which itself inhibits TSH secretion $[4,20]$.

Subclinical hypothyroidism is important and has serious long-term complications such as cardiovascular problems. Although only severe subclinical hypothyroidism (TSH more than $10 \mu \mathrm{IU} / \mathrm{ml}$ ) requires urgent treatment, we need to follow-up patients with mild and moderate subclinical hypothyroidism (TSH 4-10 $\mu \mathrm{IU} / \mathrm{ml}$ ) and closely monitor clinical signs and symptoms of overt hypothyroidism in them [12-14]. In our study, only one boy (14 years old, BMI 21.05) had grade 3 subclinical hypothyroidism (TSH $12.3 \mu \mathrm{IU} / \mathrm{ml}$ ). He had juvenile myoc- 
lonic epilepsy, and our endocrinologist decided to treat him by levothyroxine.

Our results should be interpreted in the face of certain limits. We did not assess baseline thyroid function in our children before treatment by anticonvulsive agents; however, we had a group of healthy controls to compare subclinical hypothyroidism in the normal population. We also did not follow thyroid function in children who were candidates for drug withdrawal. Another study showed that after withdrawal of anticonvulsive medications thyroid function disturbances are reversible [11]. We did not measure serum levels of anticonvulsive medications to correlate these serum levels by thyroid function tests; therefore, we could not define a correlation between serum levels of these anticonvulsive medications and grades of subclinical hypothyroidism in our patients.

\section{CONCLUSIONS}

In conclusion, our results indicate that in children with epilepsy who were treated by either carbamazepine, sodium valproate, or phenobarbital as monotherapy, subclinical hypothyroidism could occur and is relatively common (about 20\%). Our results indicate that monitoring of thyroid function using T4 and TSH serum levels should be considered in these children during anticonvulsive therapy. However, understanding the long-term effects of anticonvulsive medications on thyroid function requires well-designed, prolonged cohort studies.

\section{ACKNOWLEDGMENTS}

This paper is from the thesis of Ashraf Sepehran, MD, registration number D/884. The Research Deputy of Ahvaz Jundishapur University of Medical Sciences provided financial and logistic support for this study (grant no. U/92193) but had no role in the study design; data collection, analysis, or interpretation; writing the report; or in the decision to submit the article for publication. The authors are grateful to all parents and patients for their participation in this study. We are extremely indebted to the authorities of the Research Deputy of Ahvaz Jundishapur University of Medical Sciences for their financial and logistic support.

\section{DISCLOSURE}

The authors declare no conflict of interest.

\section{REFERENCES}

1. Aggarwal A, Rastogi N, Mittal H, et al. Thyroid hormone levels in children receiving carbamazepine or valproate. Pediatr Neurol 2011; 45: 159-162.
2. Caksen H, Dulger H, Cesur Y, et al. Evaluation of thyroid and parathyroid functions in children receiving long-term carbamazepine therapy. Int J Neurosci 2003; 113: 1213-1217.

3. Cansu A, Serdaroglu A, Camurdan O, et al. The evaluation of thyroid functions, thyroid antibodies, and thyroid volumes in children with epilepsy during short-term administration of oxcarbazepine and valproate. Epilepsia 2006; 47: 1855-1859.

4. Eiris-Punal J, Del Rio-Garma M, Del Rio-Garma MC, et al. Longterm treatment of children with epilepsy with valproate or carbamazepine may cause subclinical hypothyroidism. Epilepsia 1999; 40: 1761-1766.

5. Hirfanoglu T, Serdaroglu A, Camurdan O, et al. Thyroid function and volume in epileptic children using carbamazepine, oxcarbazepine and valproate. Pediatr Int 2007; 49: 822-826.

6. Isojarvi JI, Turkka J, Pakarinen AJ, et al. Thyroid function in men taking carbamazepine, oxcarbazepine, or valproate for epilepsy. Epilepsia 2001; 42: 930-934.

7. Kafadar I, Kilic BA, Arapoglu M, et al. Evaluation of thyroid hormones in children receiving carbamazepine or valproate: a prospective study. J Child Neurol 2015; 30: 63-68.

8. Kim SH, Chung HR, Kim SH, et al. Subclinical hypothyroidism during valproic acid therapy in children and adolescents with epilepsy. Neuropediatrics 2012; 43: 135-139.

9. Sahu JK, Gulati S, Kabra M, et al. Evaluation of subclinical hypothyroidism in ambulatory children with controlled epilepsy on valproate monotherapy. J Child Neurol 2012; 27: 594-597.

10. Turan MI, Cayir A, Ozden O, Tan H. An examination of the mutual effects of valproic acid, carbamazepine, and phenobarbital on 25-hydroxyvitamin D levels and thyroid function tests. Neuropediatrics 2014; 45: 16-21.

11. Vainionpaa LK, Mikkonen K, Rattya J, et al. Thyroid function in girls with epilepsy with carbamazepine, oxcarbazepine, or valproate monotherapy and after withdrawal of medication. Epilepsia 2004; 45: 197-203.

12. Adlin V. Subclinical hypothyroidism: deciding when to treat. Am Fam Physician 1998; 57: 776-780.

13. Javed Z, Sathyapalan T. Levothyroxine treatment of mild subclinical hypothyroidism: a review of potential risks and benefits. Ther Adv Endocrinol Metab 2016; 7: 12-23.

14. Pearce SH, Brabant G, Duntas LH, et al. 2013 ETA Guideline: Management of Subclinical Hypothyroidism. Eur Thyroid J 2013; 2: 215-228.

15. Proposal for revised classification of epilepsies and epileptic syndromes. Commission on Classification and Terminology of the International League Against Epilepsy. Epilepsia 1989; 30: 389-399.

16. Jones RM, Butler JA, Thomas VA, et al. Adherence to treatment in patients with epilepsy: associations with seizure control and illness beliefs. Seizure 2006; 15: 504-508.

17. Mansourian AR, Ahmadi AR, Saifi A, Bakhshandehnosrat S. The children reference range of thyroid hormones in Northern Iran. Pak J Biol Sci 2010; 13: 862-865.

18. Verrotti A, Basciani F, Morresi S, et al. Thyroid hormones in epileptic children receiving carbamazepine and valproic acid. Pediatr Neurol 2001; 25: 43-46.

19. Verrotti A, Laus M, Scardapane A, et al. Thyroid hormones in children with epilepsy during long-term administration of carbamazepine and valproate. Eur J Endocrinol 2009; 160: 81-86.

20. Loscher W, Schmidt D. Increase of human plasma GABA by sodium valproate. Epilepsia 1980; 21: 611-615. 